\title{
Metodología para la detección de fallas en una estructura entramada metálica empleando las técnicas de análisis modal y PSO
}

\section{Damage detection methodology in metallic structure by experimental modal analysis techniques and PSO}

\author{
Heller Sánchez-Acevedo ${ }^{1}$, Julián Uscátegui², Sergio Gómez ${ }^{3}$ \\ ${ }^{1}$ Grupo de Investigación en Energía y Medio Ambiente - GIEMA, Escuela de Ingeniería Mecánica, Universidad Industrial de \\ Santander, Colombia. Email: hgsanche@uis.edu.co \\ ${ }^{2}$ Escuela de Ingeniería Mecánica, Universidad Industrial de Santander, Colombia. Email: uscategui1528@gmail.com \\ ${ }^{3}$ Industrias Hermes Acevedo Muñoz, Colombia. Email: seedgomez@gmail.com
}

RECIBIDO: Agosto 19, 2016. ACEPTADO: Marzo 20, 2017. Versión FinAL: Mayo 30, 2017.

\begin{abstract}
RESUMEN
Con el objetico de mejorar la confiabilidad de los sistemas productivos, las empresas han desarrollado metodologías para la detección de fallos incipientes. Las estructuras metálicas son revisadas con un procedimiento de inspección visual el cual no permite la detección de fallos emergentes. El propósito del presente trabajo es desarrollar una nueva metodología, basada en el análisis modal y en un algoritmo de optimización por enjambre de partículas, para detectar fallas en estas estructuras. Un modelo físico fue construido y este fue estudiado aplicando las técnicas de análisis modal. Un modelo numérico fue establecido por medio del Método de los Elementos Finitos. El algoritmo fue ejecutado y los resultados fueron comparados con resultados experimentales. La metodología fue validada y daños de diferentes niveles fueron identificados.
\end{abstract}

PALABRAS CLAVE: Análisis modal, Algoritmo de optimización, Caracterización dinámica, Técnicas de identificación de daño basada en vibraciones (TIDBV), Métodos de elementos finitos.

\begin{abstract}
With the objective of improving the production systems reliability the companies have developed methodologies to detect incipient damages. The metallic structures are usually checked by visual inspection procedures which are not able to detect emerging falls. The purpose of the present work is to develop a novel methodology based on modal testing analysis and particle swarm optimization algorithm to detect failure in these structures. A physical model was built and it was studied applying the modal testing techniques. A numerical model was stablished by Finite Element Method (FEM). The algorithm was executed, and the results were compared with the experimental results. The methodology was validated and failure was identified for different levels damage.
\end{abstract}

KEYWORDS: Modal analysis, Optimization algorithm, Dynamic characterization, Vibration based damage identification techniques (VBDIT), Finite element method.

\section{INTRODUCCIÓN}

En la actualidad, la alta competitividad de los mercados ha obligado a las empresas a tomar decisiones importantes que les orientan, entre otras cosas, hacia la optimización de sus sistemas y procesos productivos, mejorando la calidad de los productos, reduciendo los costos y mejorando los rendimientos financieros, incluyendo esto la mejora de sus índices de confiabilidad. Enfocados en esta necesidad, se han desarrollado diversos métodos de diagnóstico de fallas, que permiten la detección temprana, el aseguramiento de la operación y la prolongación de la vida útil del sistema. Estas estrategias han sido ampliamente empleadas en todas las

Este artículo puede compartirse bajo la licencia CC BY-ND 4.0 y se referencia usando el siguiente formato: H. G. Sánchez, J. Uscátegui, S. Gómez, "Metodología para la detección de fallas en una estructura entramada metálica empleando las técnicas de análisis modal y PSO", UIS Ingenierías, vol. 16, no. 2, pp. 43-50, Julio-Diciembre 2017. Doi: https://doi.org/10.18273/revuin.v16n2-2017004 
áreas de la ingeniería, como las desarrolladas por el autor en rotodinámica [1] y las primeras incursiones en estructuras [2].

Existen diferentes métodos de detección de daños no destructivos (DDND), aplicables también para el caso de las estructuras entramadas metálicas objeto de estudio del presente trabajo, tales como la inspección visual, la inspección acústica, el ultra sonido e incluso algunos métodos térmicos [3][4], que apuntan a la consecución del objetivo empresarial. Todos ellos realizan una detección de daño local y responden satisfactoriamente en su espectro de aplicación. No obstante, presentan la desventaja de que estos métodos requieren conocer la posible localización del daño. Es por ello que, investigaciones como la presente, se enfocan en técnicas alternativas que permitan realizar una búsqueda en toda la estructura para detectar el fallo, ubicarlo y cuantificar su magnitud, sin inspeccionar de forma independiente miembro a miembro.

\section{PLANTEAMIENTO DEL PROBLEMA}

Las técnicas de inspección de estructuras, ampliamente conocidas, se basan en la revisión independiente de cada uno de los miembros que la constituyen. Esta tarea, como ha de esperarse, se hace tediosa y costosa. Por lo tanto, es necesario incursionar en la implementación de nuevos métodos que permitan la detección de fallas de forma más eficiente. Métodos que por un lado reduzcan los tiempos y los costos del proceso, y por otro que sean efectivos.

El modelo de detección de falla, empleado para el desarrollo de esta investigación se fundamenta en que, la presencia de un daño en cualquier miembro de la estructura se evidencia en la variación de las propiedades modales de la misma, producto de la pérdida de rigidez en el elemento afectado. Es por ello que, el problema se enfoca en implementación de un método que permita la detección de esas pequeñas variaciones de las propiedades modales, que las traduzca en valores que puedan ser interpretados para determinar tanto la presencia del fallo como su ubicación.

\section{MODELO}

\subsection{Análisis modal}

El modelo para la detección de falla se fundamenta en la técnica de análisis modal, tanto teórica como experimental. Esta técnica tiene por objeto la determinación de las frecuencias naturales, modos de vibración y amortiguamiento modales del sistema [5][6]. Desde el punto de vista experimental, llamado Análisis Modal Experimental (EMA), la técnica busca relacionar la respuesta vibratoria del sistema con las fuerzas de excitación que las genera, y ha sido ampliamente utilizada para la caracterización modal de elementos de máquinas, placas, etc. [7][8][9]. En este caso, tanto las amplitudes de vibración como las características de la fuerza aplicada son conocidas. La excitación puede ser aplicada con un hammer o con un excitador electrodinámico. Este último es el empleado en el presente trabajo, mientras que la señal de vibración es obtenida mediante acelerómetros.

\subsection{Modelo numérico de la estructura}

El modelo desarrollado considera un sistema de vibración libre no amortiguada, donde el conjunto de ecuaciones se define por la siguiente expresión:

$$
m \ddot{x}+k x=0
$$

ecuación en la que $m$ es la masa, $k$ es la constante de elasticidad y $x$ es el desplazamiento del sistema en función del tiempo. La amplitud de la respuesta, en el tiempo $(t)$, está dada por:

$$
x(t)=A \sin (\omega t+B), \quad \omega_{n}=\sqrt{k / m}
$$

$A$ y $B$ son constantes reales y $\omega_{n}$ representa la frecuencia natural del sistema. La ecuación (1) se puede expresar de forma más generalizada respecto al número de grados de libertad como sigue:

$$
[M] \ddot{x}(t)+[K] x(t)=0
$$

donde $M$ es la matriz de masa, $K$ la matriz de rigidez y $x$ es el desplazamiento en los $n$ grados de libertad que posea el sistema [2].

Tanto la matriz de masas como la matriz de rigidez son planteadas a partir de las propiedades fisico mecánicas del material y de la geometría de los elementos y de la estructura. La topología de la estructura objeto de estudio es representada en la Figura 1.
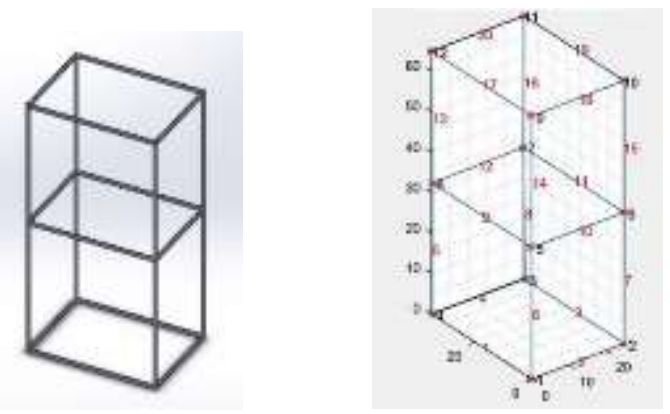

Figura 1. Topología de la estructura en MATLAB. 
Las distribuciones de datos de dichas matrices se pueden apreciar en la Figura 2. Esta, corrobora la distribución esperada de dicha información.
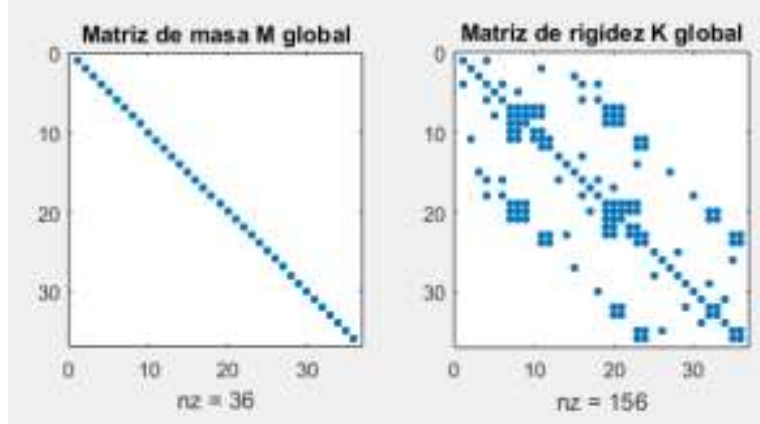

Figura 2. Distribución de datos de la matriz global de masa y rigidez respectivamente.

La solución del sistema de ecuaciones es implementada en MatLab. Este proceso retorna la información modal de una estructura, cuya condición se fija como referencia. Este modelo numérico debe ser validado contra su modelo físico.

\subsection{Inclusión de ruido}

El modelo considera la introducción aleatoria de perturbaciones en los datos, de las frecuencias y formas modales, con el objeto de buscar una mayor coherencia entre la respuesta dinámica del modelo numérico en relación con el modelo físico. Estas perturbaciones han sido incluidas en el modelo a través de las siguientes expresiones:

$$
\begin{aligned}
& \omega_{\mathrm{r}}=\omega *(1+\operatorname{rand}(-1,1) * \text { Ruido } \omega) \\
& \emptyset_{\mathrm{r}}=\emptyset *(1+\operatorname{rand}(-1,1) * \text { Ruido } \emptyset)
\end{aligned}
$$

donde $\omega$ y $\emptyset$ corresponden a las frecuencias naturales y a las formas modales originales, respectivamente; $\omega_{\mathrm{r}}$ y $\emptyset_{\mathrm{r}}$ corresponden a los valores con la inclusión del ruido; Ruidow y Ruido $\emptyset$ son los porcentajes de ruido incluidos en el modelo. Farrar y Cone [10], reportan en su trabajo que, usualmente las frecuencias naturales $y$ formas modales son contaminadas por ruido con un error promedio de $\pm 0.15 \%$ para Ruidow y $\pm 3.0 \%$ para Ruido $\emptyset$.

\subsection{Inclusión del daño}

Las variaciones en los parámetros dinámicos de una estructura están ligadas en su mayoría a las pérdidas de rigidez de determinado elemento, producto de diferentes problemas como la corrosión y fallos en los elementos de sujeción. Para incluirle un daño en el modelo se ha planteado un coeficiente de pérdida de rigidez $\beta$. Este valor varía entre 0 y 0.6 , donde 0 quiere decir que no hay presencia de daño y 0.6 es pérdida total del elemento, solo en estado lineal. Se ha empleado como estrategia la disminución del módulo de elasticidad para hacer efectiva la pérdida de rigidez en un elemento. El efecto se introduce de la siguiente forma:

$$
E_{D}=E_{S} *(1-\beta)
$$

donde $E_{D}$ es el módulo de elasticidad reducido para provocar el daño y $E_{S}$ es el módulo de elasticidad del material [11]. Otra forma empleada para la inclusión de daño en la estructura es la variación del área, donde se utiliza el mismo principio de la ecuación (6).

\subsection{Modelo físico}

Se establece una estructura entramada metálica, ver Figura 1, compuesta por 20 barras de acero AISI SAE 1020, con un diámetro de 9,8 [mm] soldadas entre sí. Tiene forma simétrica $y$ un tamaño medio con dimensiones de 350 [mm] x 250 [mm] y una altura de 650 [mm]. Las propiedades del material han sido definidas de la siguiente forma: densidad $\rho=7850$ $\left[\mathrm{Kg} / \mathrm{m}^{\wedge} 3\right]$, módulo de elasticidad $\mathrm{E}=2.1 \mathrm{E} 11[\mathrm{~Pa}] \mathrm{y}$ relación de Piosson $v=0.3$.

Sobre este modelo se generan tres escenarios diferentes de falla, tres niveles de severidad. De cada uno de ellos se obtiene la información modal de forma experimental. Esto datos se emplean para la sintonización y validación del modelo de detección de falla.

En el primer escenario se realiza un corte en el centro de la barra superior con una profundidad de 1/4 del diámetro de la misma; en el segundo escenario se profundiza el mismo corte hasta $1 / 2$ del diámetro de la barra; por último, en el tercer escenario, se profundiza aún más el corte hasta llegar a un valor de 3/4 del diámetro de la barra. Las características generales de los fallos generados se aprecian en la Figura 3.

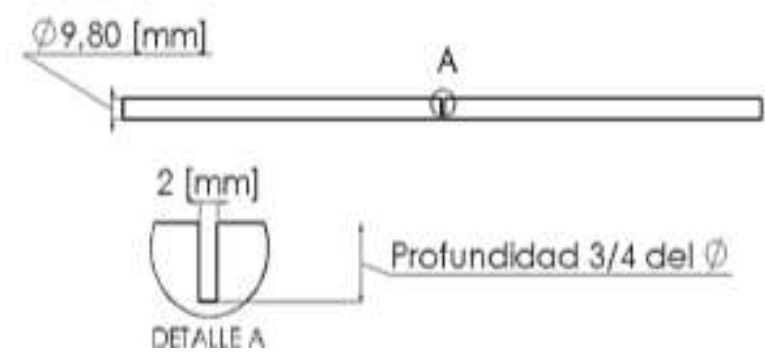

Figura 3. Vista en detalle del corte inducido en la barra superior. Daño considerado como grave. 


\section{MONTAJE EXPERIMENTAL}

La obtención de las propiedades modales de la estructura se realiza mediante el EMA. La excitación se aplica en un punto fijo, empleando un shaker. La respuesta vibratoria de la estructura se obtiene midiendo con acelerómetros en diferentes puntos de la misma. El montaje del sistema completo se puede apreciar en la Figura 4.

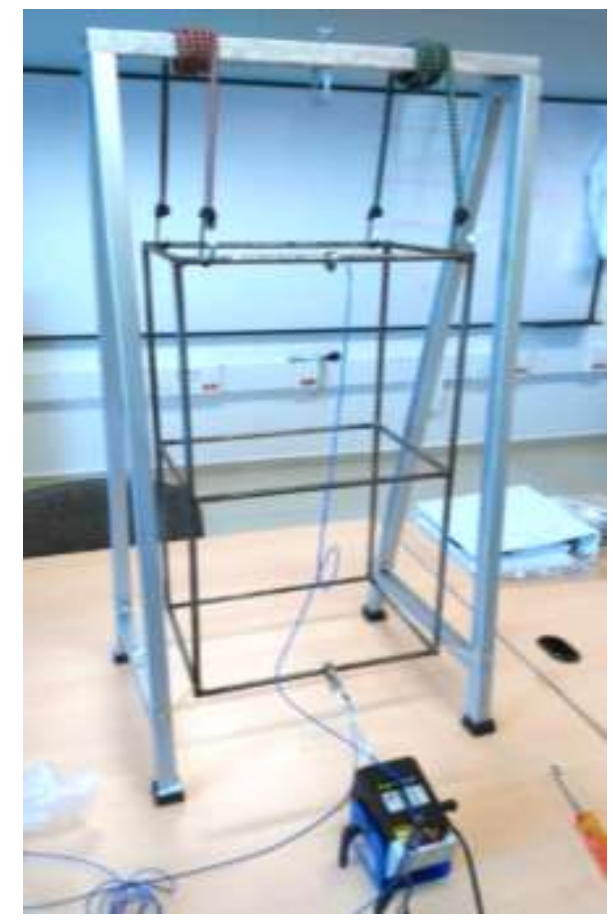

Figura 4. Estructura soporte con bandas elásticas.

La adquisición de datos, el tratamiento digital de la señal y la obtención de las Funciones de Respuesta en Frecuencia (FRFs), se realiza empleando una tarjeta de adquisición de la National Instrument y el módulo Sound \& Vibration de LabView, ver Figura 5. Para este ensayo, se empleó como señal vibratoria del excitador electrodinámico ruido blanco.

Los datos experimentales permiten: por una parte, obtener la información de referencia de la estructura, que corresponde a la caracterización de la misma en condiciones adecuadas de operación, para compararla con la información extraída en condición de daño inducido. Por otra, sintonizar y validar el modelo numérico, de tal forma que sus resultados se correspondan con lo que está ocurriendo en el modelo físico. Ver Tabla 1. El objetivo final es, emplear estos datos fiables como insumo para el modelo de detección de falla.
Tabla 1. Resultado de caracterización modal proceso numérico vs proceso experimental.

\begin{tabular}{c|ccc} 
Modo & $\begin{array}{r}\text { EMA } \\
(\mathrm{Hz})\end{array}$ & $\begin{array}{c}\text { Frecuencias } \\
\text { Matlab }(\mathrm{Hz})\end{array}$ & $\begin{array}{c}\text { Porcentaje } \\
\text { de error } \\
{[\%]}\end{array}$ \\
\hline 1 & 0,0009 & 0 & \\
2 & 49,687 & 45,6453 & 0,09 \\
3 & 59,285 & 65,67734 & 3,88 \\
4 & 74,419 & 73,8647 & 0,75 \\
5 & 76,746 & 80,97388 & 5,22 \\
6 & 80,764 & 83,2647 & 3 \\
7 & 82,637 & 83,7354 & 1,31 \\
8 & 92,057 & 93,8464 & 1,91
\end{tabular}

\section{MODELO DE DETECCIÓN DE FALLA BASADO EN PSO}

El modelo se fundamenta en la identificación de las diferencias encontradas en la caracterización modal de la estructura a evaluar, respecto de un estado de referencia de la misma estructura, condición de la estructura definida como "estructura sana".

Para realizar el proceso de búsqueda se ha implementado un algoritmo de optimización PSO (Particle Swarm Optimization). En este proceso se correlaciona la información modal de la estructura obtenida de forma experimental, con la respuesta numérica de un modelo, en principio validado. La búsqueda se centra en encontrar un modelo que, variando el módulo de elasticidad de uno o varios elementos, tenga un comportamiento similar al modelo físico. Para ello emplea como función objetivo la planteada en la Ecuación 7, que minimiza la diferencia, bien podría llamarse el error, entre la información experimental y el modelo numérico.

$$
F=\sum_{j=1}^{r} a_{f}\left[1-\left(\frac{W_{j}^{m}}{W_{j}^{a}}\right)\right]^{2}+\sum_{t=1}^{s} \sum_{j=1}^{r} b_{t j}\left(\emptyset_{t j}^{m}-\emptyset_{t j}^{a}\right)
$$

En la expresión anterior, el superíndice $m$ hace referencia a los datos que fueron obtenidos experimentalmente y el superíndice $a$ para aquellos obtenidos a partir del modelo numérico. Otros factores como $W_{j}$ y $\emptyset_{j}$, corresponden jésima frecuencia natural y forma modal, respetivamente. Los términos $a$ y $b$ son factores de peso para cada una de las características dinámicas, consideradas iguales en el presente trabajo. El término $r$ es el número de formas modales utilizadas para la comparación y el término $s$ es el número de componentes del vector de la j-ésima forma modal [12]. 


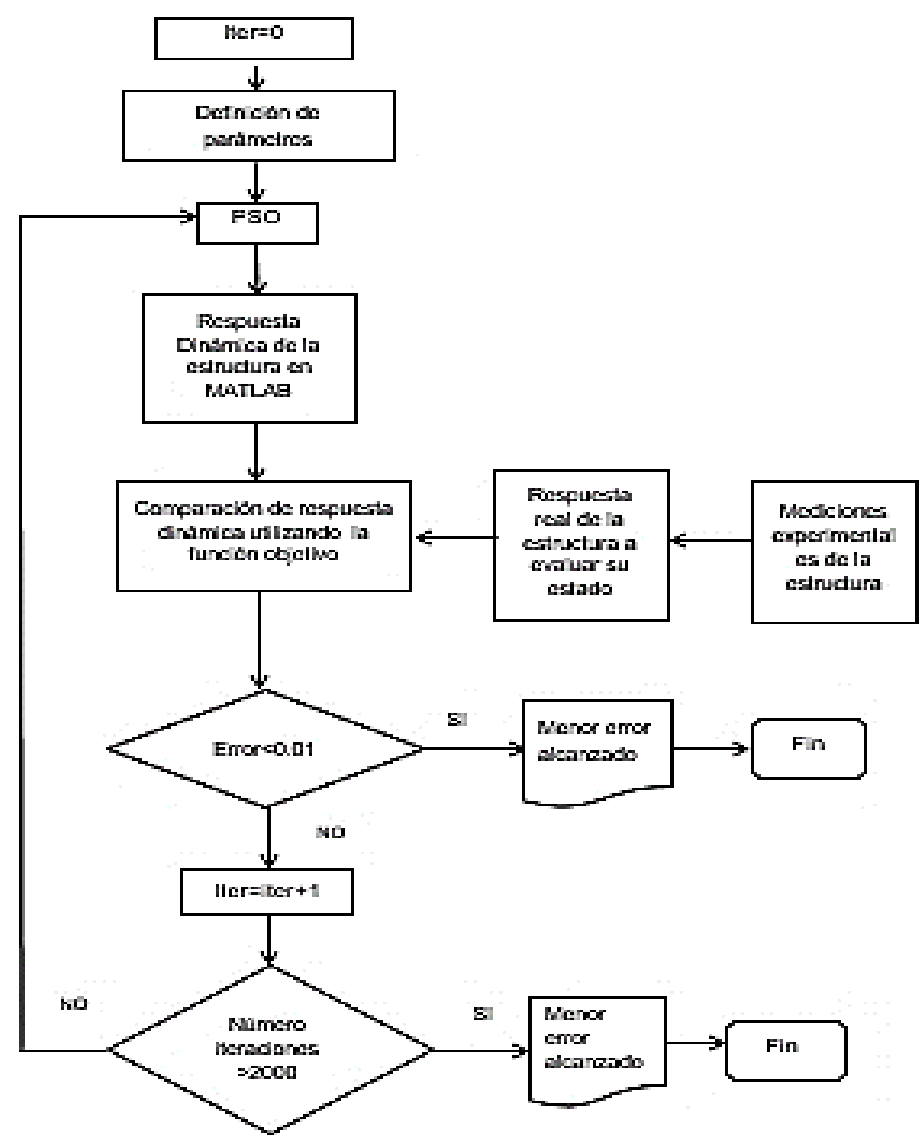

Figura 6. Modelo del proceso de identificación de daño basado en PSO.

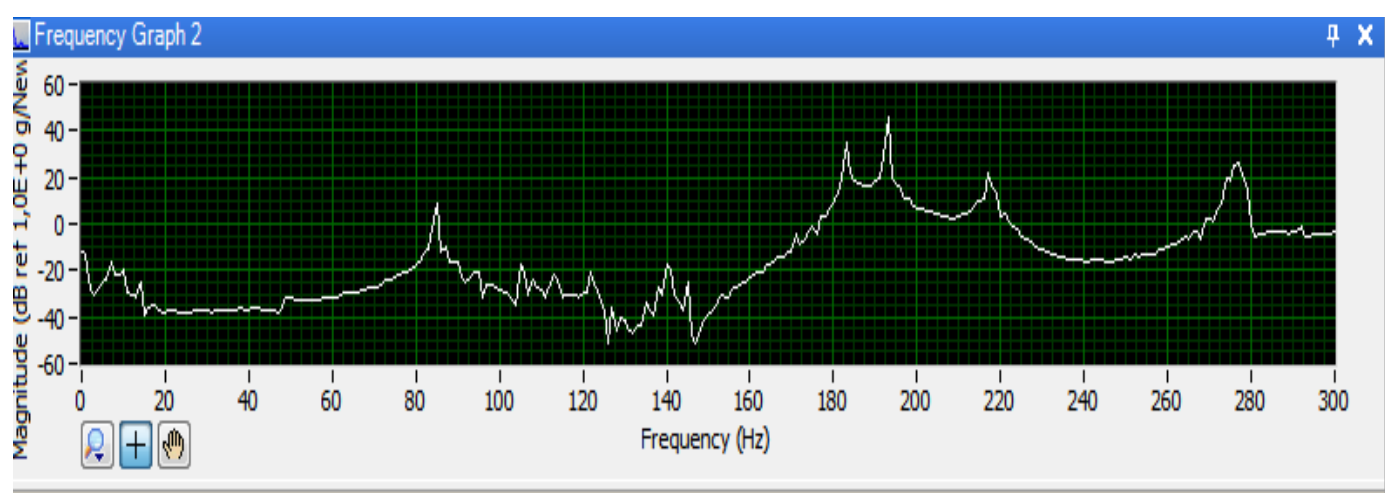

Frequency Graph 3

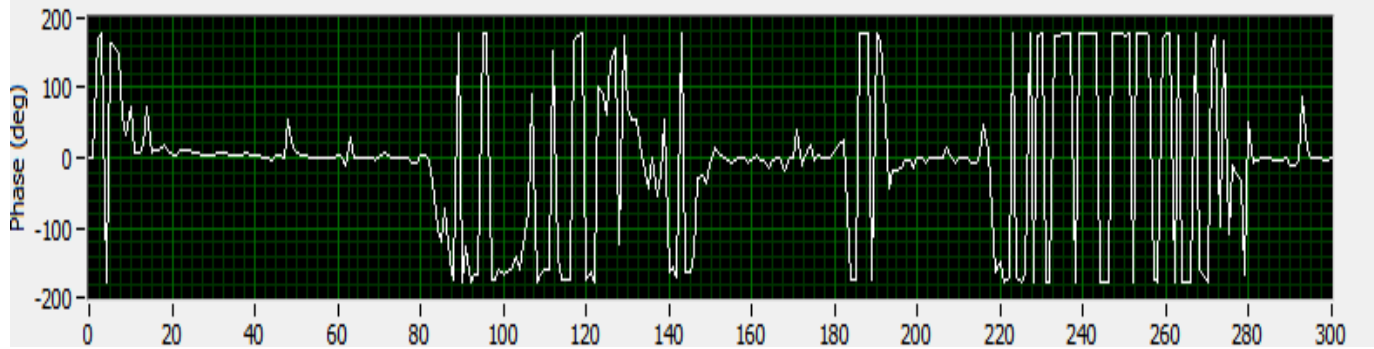

Figura 5. Funciones de Respuesta en Frecuencia de la estructura. 
En la Figura 6, se puede apreciar el diagrama de flujo que hace referencia, en términos generales, al proceso seguido para la detección de falla. Es de resaltar que, el modelo numérico se ejecuta en cada iteración para recalcular las frecuencias naturales y formas modales con la nueva distribución de rigidez. La pérdida de rigidez está asociada con la presencia de un fallo.

\section{RESULTADOS DE EVALUACIÓN Y APLICACIÓN DEL MODELO}

El modelo es ejecutado con la información experimental obtenida del Análisis Modal aplicado al modelo físico descrito anteriormente. Para el algoritmo de búsqueda se ha empleado un número máximo de 200 individuos, aceleración en un rango entre 0.6 y 4.7 , factores de inercia entre 0.4 y 0.6 y un factor de estrechamiento de 0.729 .

\subsection{Comportamiento del algoritmo respecto al ruido}

Como es bien sabido, la inclusión de ruido en el modelo teórico busca la proximidad con el modelo experimental. Por tanto, se realiza un análisis del comportamiento de las iteraciones respecto a diferentes niveles de ruido y, además, se determina el costo computacional que tiene la convergencia del algoritmo.

En la Figura 7 se midió el tiempo que tarda en converger el algoritmo con un ruido de $0.15 \%$ en las frecuencias naturales y de $3 \%$ para las formas modales.

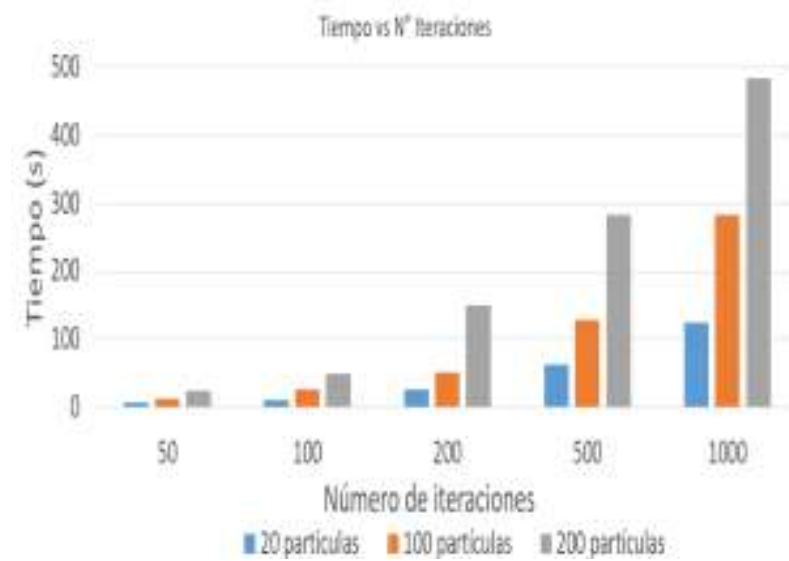

Figura 7. Gráfica de tiempo vs número de iteraciones.

Por otra parte, en la Figura 8 se muestra la efectividad del algoritmo para dar solución al problema, respecto a diferentes niveles de ruido y diferentes cantidades de individuos en la población del enjambre. El análisis permite establecer los niveles de ruido que pueden ser tenidos en cuenta para aplicar el algoritmo en el proceso de detección de falla de forma estable.
EFECTIVIDAD OEL ALGORITMO

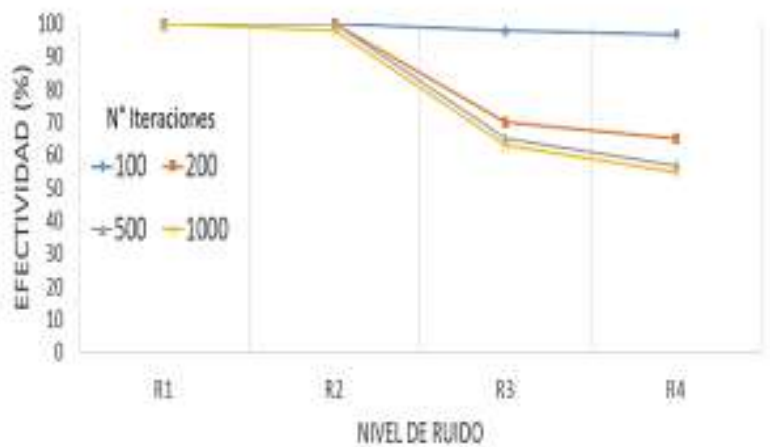

Figura 8. Gráfica del porcentaje de efectividad de la detección del daño en función del número de iteraciones y el nivel de ruido, donde $\mathrm{R}=[$ Ruido $\omega$, Ruido $\Phi]: \mathrm{R} 1=[0 \%, 0 \%], \mathrm{R} 2=$ $[0.1 \%, 1 \%], \mathrm{R} 3=[0.15 \%, 3 \%], \mathrm{R} 4=[0.2 \%, 4 \%]$.

\subsection{Mínimo nivel de daño}

Este análisis tiene por objeto determinar el mínimo nivel de daño al que es sensible el modelo. La Figura 9 muestra un barrido de diferentes longitudes de corte, escenarios de daño aplicados a una barra del modelo físico. Se establecen 8 escenarios de daño haciendo cortes de diferente profundidad, de la siguiente forma: $\mathrm{D} 1=3 / 4$, D2 $=1 / 2$, D3=1/4, D4=1/8, D5=1/16, D6=1/32, D7=1/64 y $\mathrm{D} 8=1 / 132$, donde cada escenario se refiere a la fracción de reducción del área hecho sobre la sección transversal de la barra. Se ejecuta el algoritmo de detección de falla considerando porcentajes de ruido de $0.15 \%$ para las frecuencias y de $3 \%$ para las formas modales.

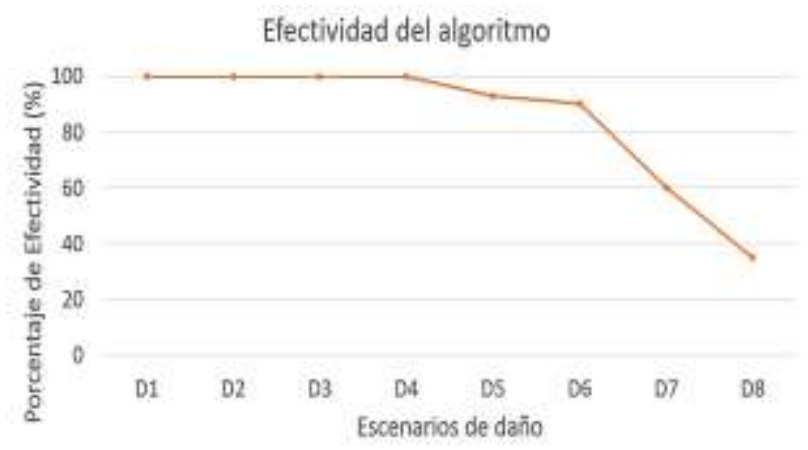

Figura 9. Gráfica de mínimo nivel de daño obtenido.

Se puede apreciar en la Figura 9 que, el modelo responde adecuadamente hasta reducciones de 1/32 de área.

\subsection{Detección de fallos inducidos}

Para el caso del daño, con afectación del área en D1=3/4, en la barra 17, los resultados son presentados en la Figura 10. El algoritmo ha sido ejecutado sin ruido y con niveles de ruido de $0.15 \%$ para las frecuencias y $3 \%$ para las 
formas modales. En la Figura 10 se aprecian los resultados comparativos de la información del caso real vs los resultados numéricos producto de la ejecución del algoritmo.

Para verificar la respuesta del algoritmo ante la afectación de otra barra, seleccionada de forma aleatoria, se procede a inducir el fallo, bajo el escenario D1=3/4, teniendo en cuenta los mismos niveles de ruido, $0.15 \%$ para frecuencias y $3 \%$ para las formas modales. Los resultados de la ejecución del algoritmo se presentan en la Figura 11.

\section{CONCLUSIONES}

Se ha implementado un algoritmo, basado en PSO y en las técnicas de análisis modal, que permite la detección de fallos en una estructura metálica.

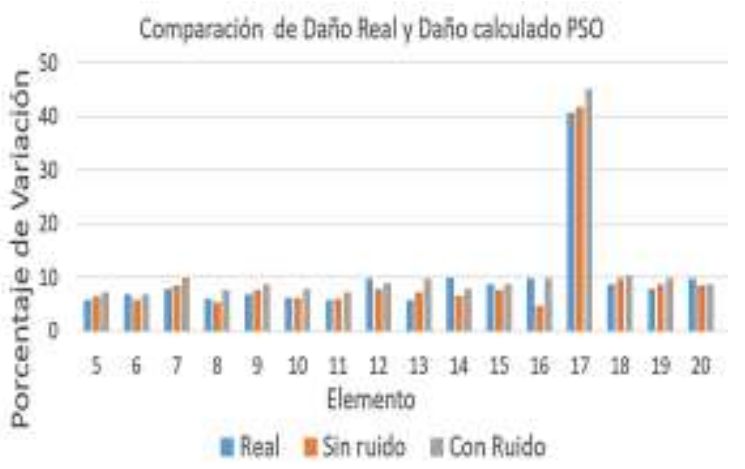

Figura 10. Porcentaje de Daño real y Daño calculado PSO, con ruido y sin ruido, en escenario de daño grave, modo $6 \mathrm{de}$ vibración.

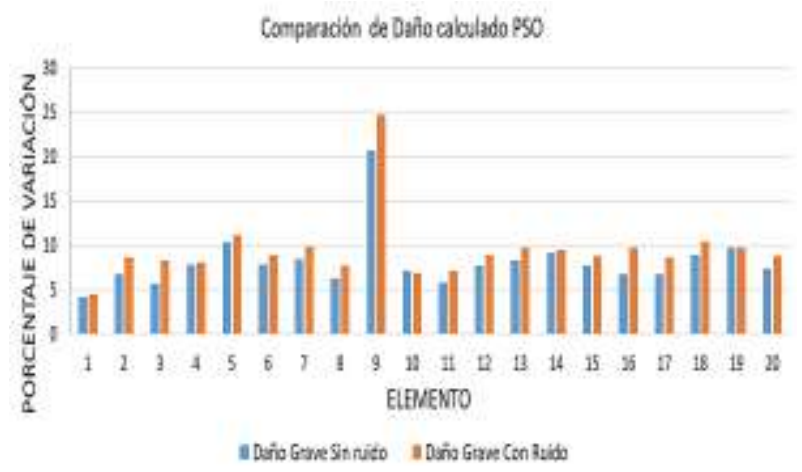

Figura 11. Porcentaje de Daño calculado PSO, con ruido y sin ruido, en escenario de daño grave, modo 6 de vibración.

Otro escenario posible es la presencia de más de un fallo en la estructura. Es decir, podría presentarse que dos o más barras generaran pérdida de rigidez por la presencia de un daño en las mimas. El algoritmo ha sido ejecutado con daños inducidos en dos barras, con un nivel de afectación $\mathrm{D}=1 / 2$. Los resultados son presentados en la Figura 12.

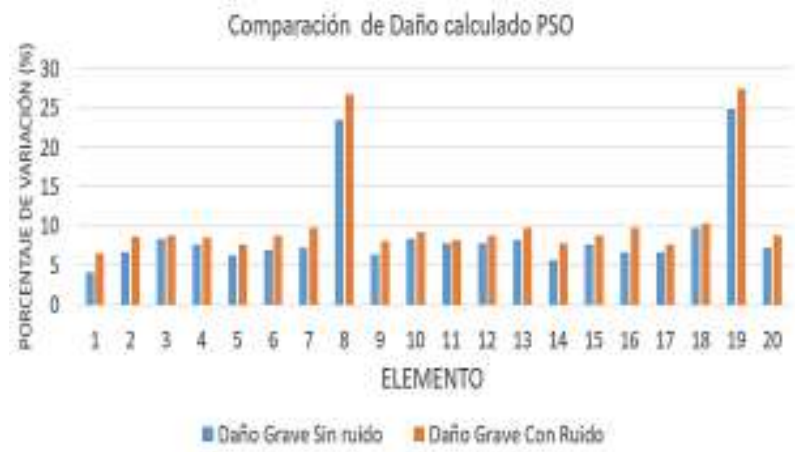

Figura 12. Porcentaje de Daño calculado PSO, con ruido y sin ruido, en escenario de daño grave, modo 6 de vibración, con dos elementos dañados.

El algoritmo ha sido contrastado con información experimental y los resultados han sido satisfactorios.

Diferentes niveles de daño han sido inducidos en el modelo y se ha registrado su comportamiento. Los datos indican que el modelo es sensible a un nivel de fallo determinado por la reducción de 1/32 del área de la sección transversal de una barra.

Con el objeto de acercar el algoritmo un poco más al comportamiento real de la estructura, se han introducido diferentes niveles de ruido. Este ejercicio ha permitido registrar que, el algoritmo mantiene buen comportamiento hasta un nivel máximo de error del $0.15 \%$ en frecuencia y $3 \%$ en las formas modales.

\section{REFERENCIAS}

[1] H. G. Sánchez A., "Modelo analítico para el estudio de rotores flexibles," Rev. UIS Ing., vol. 9, no. 1, pp. 6976, Jun. 2010.

[2] J. M. Pachón, O. A. González, and H. G. Sánchez, "Detección de daños en una armadura unidimensional por medio del algoritmo de optimización de la luciérnaga y elementos finitos," Av. Investig. en Ing., vol. 13, no. 1, pp. 1-7, 2017.

[3] A. Katunin, K. Dragan, and M. Dziendzikowski, "Damage identification in aircraft composite structures: A case study using various non-destructive testing techniques," Compos. Struct., vol. 127, pp. 1-9, Sep. 2015.

[4] M. P. Paulraj, S. Yaacob, M. S. A. Majid, M. N. F. M. Kazim, and P. Krishnan, "Structural Steel Plate Damage Detection using Non Destructive Testing, Frame 
Energy based Statistical Features and Artificial Neural Networks," Procedia Eng., vol. 53, pp. 376-386, 2013.

[5] D.-J. Han, "Generalized modal balancing for nonisotropic rotor systems," Mech. Syst. Signal Process., vol. 21, no. 5, pp. 2137-2160, 2007.

[6] S. Živanović, A. Pavic, and P. Reynolds, "Modal testing and FE model tuning of a lively footbridge structure," Eng. Struct., vol. 28, no. 6, pp. 857-868, 2006.

[7] A. delli Carri, B. Weekes, D. Di Maio, and D. J. Ewins, "Extending modal testing technology for model validation of engineering structures with sparse nonlinearities: A first case study," Mech. Syst. Signal Process., vol. 84, pp. 97-115, 2017.

[8] G. Mansour, K. Tsongas, and D. Tzetzis, "Modal testing of nanocomposite materials through an optimization algorithm," Measurement, vol. 91, pp. 3138, 2016.

[9] A. D. Nembhard and J. K. Sinha, "Comparison of experimental observations in rotating machines with simple mathematical simulations," Measurement, vol. 89, pp. 120-136, 2016.

[10] C. R. Farrar and S. W. Doebling, "An overview of modal based damage identification methods," Struct. Damage Assess. Using Adv. Signal Process. Proced., 1997.

[11] K.-C. Chang and C.-W. Kim, "Modal-parameter identification and vibration-based damage detection of a damaged steel truss bridge," Eng. Struct., vol. 122, pp. 156-173, Sep. 2016.

[12] K. Moslem and R. Nafaspour, "Structural Damage Detection by Genetic Algorithms," AIAA J., vol. 40, no. 7, pp. 1395-1401, Jul. 2002. 